\title{
Frequency Distribution of Pollen Types in Honey Samples of Apiscerana F. Collected from Different Areas of Shiwalik Hills
}

\author{
Avinash Kaur, V. K. Mattu \\ Sociobiology and Behavioral Ecology Research Lab \\ Department of Biosciences, Himachal Pradesh University, Shimla (H.P)-171005, India
}

\begin{abstract}
Most of the flowering plants are pollinated by insects and many insects are specific to certain species of plants. Honey bees; on the other hand have no such restriction of pollen and nectar. There are at least four hundred species of plants which are either major or minor sources of pollen and nectar to Indian hive bee, ApisceranaF. It is obvious that all the plant species are not available in any one locality and a given plant species may also show variations in its utility to bees in different localities or during different years. Information on the utility of each of these plants species, its distribution, abundance and preferences for any climate or soil etc. is of great importance to beekeepers. Therefore, melissopalynological studies were conducted on twelve honey samples and pollen loads collected from different localities of Shiwalik hills. Analysis of pollen types in honey was carried using standard methods of melissopalynology. These investigations revealed that predominant sporomorphs were: Brassica sp., Raphanus sp., Murraya sp., Eucalyptus sp. etc. whereas, calendula sp., Mangiferasp, Eruca sp., Trifolium sp., Woodfordiasp.. Psidium sp., Grewia sp., Phoenix sp., Hibiscus sp., Syzygium sp., Melia sp., Grewia sp., Acacia sp., Adhatoda sp., Citrus sp., Azadirachta sp. etc. were secondary pollen types. The important minor and minor pollen components represented in these honey samples were: Acacia sp., Adhatoda sp., Ageratum sp., Allium sp., Bauhinia sp., Berberis sp., Bidens sp., Callistemon sp., Carissa sp., Cassia sp., Chenopodium sp., Dalbergia sp., Dianthus sp., Gossypium sp., Grevillea sp., Grewia sp., Helianthus sp., Lagerstroemia sp., Lilium sp., Medicago sp., Murraya sp., Rubus sp., Salvia sp., Sapindus sp., Syzygium sp., Zea sp., Zizyphus sp., Bauhinia sp., Cestrum sp., Citrus sp., Litchi sp., Raphanus sp.Bombax sp., Embilica sp., Pisum sp., Rosa sp., Woodfordia sp. Aegle sp., Albizzia sp., Berberissp., Calendula sp., Cedrella sp., Dianthus sp., Leucaenasp., Ocimum sp., Pisum sp., Polygonumsp., Punica sp., Ricinus sp., Robiniasp., Taraxacum sp., Sonchus sp., Syzygium sp., Vitis sp., Bombax sp., Litchisp and members of families Boraginaceae, Convolvulaceae, Euphorbiaceae, Fabaceae, Meliaceae, Asteraceae, CucurbitaceaeMyrtaceaeAcanthaceae, Apiaceae, Apocynaceae, Boraginaceae, Brassicaceae, Cannabinaceae, Lamiaceae, Malvaceae, Papaveraceae, Poaceae, Rosaceae, Sapindaceae and Rutaceae. Most of the honey samples had pollen of entomophilous plants with a small percentage of anemophilous types. These investigations also revealed both uniflorality and multiflorality in different honey samples. On the basis of present melissopalynological and bee botanical investigations, a floral calendar of honey plant resources of Shiwalik hills indicating their taxonomic status, geographic location, honey potentiality, periods of flowering and economic uses has been prepared. Present studies thus suggest that this region offers very rich potential for the development of beekeeping due to the multiplicity of bee flora available throughout the year. Such investigations can be helpful in setting up new apiaries, in migratory beekeeping practices and application of modern bee management technology.
\end{abstract}

Keywords: Beekeeping, Honey, Melissopalynology, Pollen, Nectar, Bee pasturase

\section{Introduction}

Insects and plants are mutually dependent upon each other. Many insects including honey bees depend upon the plants for energy to maintain their activities, whereas, plants in turn depend for pollination on insects (Seeley, 1985). This energy relationship between plants and nectar gathering insects is a necessary basis for studying the foraging behavior, crop pollination and honey production (McGregor, 1976; Free, 1993). Honey bees while foraging on the flowers of different entomophilous plants for collecting nectar; also gather some pollen with it. This pollen is retained in the ripened honey which is subsequently stored in the honey combs. The microscopical examination of these pollen grains in the honey is known as 'melissopalynology' and any final confirmatory evaluation of bee plants is incomplete without the study of melissopalynology (Deodikar, 1965; Louveauxet al., 1978; Nair, 1985; Sharma, 1989).

In view of increased application of honey pollen analysis and bee botany to apiculture, the present investigations were undertaken to determine the frequency distribution of pollen types in honey samples of Apiscerana F. collected from different areas of Shiwalik hills.

\section{Material \& Methods}

Melissopalynogical studies were conducted on different honey samples collected from various altitudes of Shiwalik hills. These hills symbolize one of the most fragile ecosystems which extend from $29^{0}-33^{0} \mathrm{~N}$ latitude to $74^{0}$ $80.5^{\circ} \mathrm{E}$ longitude. These studies were conducted by collecting a total of 12 honey samples and pollen loads form A. cerana colonies in different parts of Shiwalik hills. These collections were made mainly during the major honey flow seasons (i.e. September to October and February to June) of the years 2008 and 2009 respectively. Collection sites (and elevation in meters) are shown in Table1. Reference pollen slides of honey samples were prepared according to the method of Louveaux et al. (1978), modified by Iwama and Melhem (1979). Pollen grains recovered from the honey samples were identified with the help of reference pollen slides and counted using a haemocytometer. The absolute pollen count and percentage of pollen types were then calculated and pollen spectra constructed on the basis of those percentages. 


\section{International Journal of Science and Research (IJSR) \\ ISSN (Online): 2319-7064 \\ Index Copernicus Value (2013): 6.14 | Impact Factor (2015): 6.391}

For pollen load analysis, the pollen pellets were dispersed in water and the solution acetolysed according to the method of Erdtman (1969).

Table: 1: Frequency distribution of pollen types in honey samples of Apiscerana F. collected from different areas of Shiwalik hills of (expressed as percentage of total number of pollen grains)

\begin{tabular}{|c|c|c|c|c|c|c|c|c|c|c|c|c|}
\hline Plant species & Kumarhatti & Plasare & Ropar & Panchkula & Ramshahar & Mittian & Diggal & $\begin{array}{c}\text { Una } \\
\text { Proper }\end{array}$ & Gagret & Pinjore & Chandigarh & Derabassi \\
\hline & 1 & 2 & 3 & 4 & 5 & 6 & 7 & 8 & 9 & 10 & 11 & 12 \\
\hline Acacia sp. & - & - & - & 4.75 & - & - & - & - & - & 19.95 & - & - \\
\hline Acanthaceae & - & - & - & - & 1.95 & - & - & - & 2.89 & - & - & - \\
\hline Adhatoda sp. & - & - & 8.59 & - & - & - & - & - & - & 20.47 & - & - \\
\hline Aegle sp. & - & - & - & - & - & - & - & 1.04 & - & - & - & - \\
\hline Ageratum sp. & - & - & - & - & - & - & - & - & - & 4.15 & - & - \\
\hline Albizzia sp. & - & - & - & - & - & - & 0.98 & - & - & - & - & - \\
\hline Allium sp. & - & - & - & - & - & - & 6.85 & - & - & - & - & - \\
\hline Apiaceae & - & - & - & - & - & - & - & 2.90 & - & - & - & - \\
\hline Apocynaceae & - & - & - & - & - & - & 1.99 & - & - & - & - & - \\
\hline Asteraceae & 1.67 & 1.53 & - & - & - & 1.89 & - & 2.99 & - & - & 3.99 & 2.63 \\
\hline Azadirachta sp. & - & - & - & - & - & - & - & 16.95 & - & - & - & - \\
\hline Bauhinia sp. & - & 4.87 & - & - & - & 3.95 & 6.30 & - & - & - & - & 8.54 \\
\hline Berberis sp. & - & - & - & - & 7.74 & - & 2.83 & - & - & 2.69 & - & - \\
\hline Bidens sp. & - & - & - & - & - & - & - & 3.0 & 4.1 & - & - & - \\
\hline Bombax sp. & 3.25 & - & - & - & - & - & - & - & - & - & 0.92 & 2.09 \\
\hline Boraginaceae & - & - & - & $-1 \quad$ & - & - & - & 5.90 & - & 1.09 & - & - \\
\hline Brassica sp. & 17.58 & 16.45 & - & - & 18.59 & 45.23 & - & - & - & - & 46.06 & 30.32 \\
\hline Brassicaceae & - & - & - & - & - & - & 2.99 & - & - & - & - & - \\
\hline Calendula sp. & - & - & 16.35 & - & 2.99 & $-\quad$ & - & - & - & - & - & - \\
\hline Callistemon sp. & - & - & - & - & $1-$ & - & 8.68 & - & - & - & - & - \\
\hline Cannabinaceae & - & - & - & - & - & - & - & - & 2.89 & - & - & - \\
\hline Carissa sp. & - & - & - & - & - & - & - & - & 6.99 & - & - & - \\
\hline Cassia sp. & - & - & - & 3.93 & - & - & - & 4.30 & - & - & - & - \\
\hline Cedrella sp. & - & - & - & - & - & - & 2.98 & 9.11 & - & - & 2.50 & - \\
\hline Cestrum sp. & - & - & - & - & - & - & - & - & - & - & - & 6.48 \\
\hline Chenopodium sp. & - & - & - & $-\quad$ & 4.0 & - & - & - & - & - & - & - \\
\hline Citrus sp. & 16.86 & 8.01 & - & - & - & - & - & - & - & - & 16.01 & - \\
\hline Convolvulaceae & - & - & - & - & - & - & - & - & - & 7.94 & - & - \\
\hline Cucurbitaceae & 1.66 & 2.01 & - & - & - & 1.99 & - & - & - & - & 1.92 & - \\
\hline Dalbergia sp. & - & - & - & 5.06 & - & - & - & - & - & - & - & - \\
\hline Dianthus sp. & - & - & - & - & 0.63 & - & - & - & 3.69 & - & - & - \\
\hline Emblica sp. & 4.0 & - & 3.90 & - & - & - & - & - & - & - & 3.0 & - \\
\hline Eruca sp. & - & 16.93 & - & - & 16.73 & 16.93 & - & - & - & - & - & 35.82 \\
\hline Eucalyptus sp. & - & - & - & 47.16 & - & - & - & 16.83 & - & - & - & - \\
\hline Euphorbiaceae & - & - & - & - & - & - & - & 8.93 & - & - & - & - \\
\hline Fabaceae & - & - & 12.45 & - & - & - & - & - & 9.93 & - & - & - \\
\hline Gossypium sp. & - & - & - & - & - & - & - & \begin{tabular}{|l|}
- \\
\end{tabular} & - & 6.49 & - & - \\
\hline Grevillea sp. & - & - & - & - & - & - & 4.02 & - & - & - & - & - \\
\hline Grewia sp. & - & - & - & 16.95 & - & - & 4.69 & - & 23.99 & - & - & - \\
\hline Helianthus sp. & - & - & - & - & - & - & - & - & - & 4.69 & - & - \\
\hline Hibiscus sp. & - & - & - & -1 & - & - & 16.18 & - & - & - & - & - \\
\hline Lagerstroemia sp. & - & - & - & -11 & - & - & - & - & - & 3.05 & 3.27 & - \\
\hline Lamiaceae & - & - & - & 0.9 & - & - & - & - & - & - & - & - \\
\hline Leucaena sp. & - & - & - & - & - & - & 1.99 & - & - & - & - & - \\
\hline Lilium sp. & - & - & - & - & - & -- & - & - & 8.94 & - & - & - \\
\hline Litchi sp. & - & 3.21 & - & - & - & 4.80 & - & - & - & - & - & 2.25 \\
\hline Malvaceae & - & - & - & - & - & - & 2.98 & - & - & - & - & - \\
\hline Mangifera sp. & - & - & 21.19 & - & - & - & - & - & - & - & - & - \\
\hline Malea sp. & - & - & - & - & - & - & - & 16.71 & - & - & - & - \\
\hline Medicago sp. & - & - & - & - & - & - & - & - & - & 5.68 & - & - \\
\hline Meliaceae & - & - & 5.45 & - & - & - & - & - & - & - & 3.22 & - \\
\hline Murraya sp. & 46.15 & - & 3.96 & - & 7.98 & - & - & - & - & - & - & - \\
\hline Myrtaceae & - & - & - & - & - & - & - & - & - & 4.32 & - & - \\
\hline Ocimum sp. & - & - & - & - & - & - & - & - & - & 2.99 & - & - \\
\hline Papaveraceae & - & - & - & 0.98 & - & - & - & - & - & -- & - & - \\
\hline Phoenix sp. & - & - & - & 17.95 & - & - & - & - & - & - & - & - \\
\hline Pisum sp. & - & - & - & - & - & - & - & - & 2.09 & - & 3.06 & - \\
\hline Poaceae & - & - & - & - & - & - & - & 2.98 & - & - & - & - \\
\hline Polygonum sp. & - & - & - & - & - & - & - & - & - & 1.73 & - & - \\
\hline Psidium $s p$. & - & - & 16.12 & - & - & - & - & - & - & - & - & - \\
\hline Punica sp. & - & - & 6.43 & - & - & - & 2.69 & - & - & - & - & - \\
\hline Raphanus sp. & - & 46.99 & - & - & - & 3.89 & - & - & - & - & 16.05 & 11.87 \\
\hline Ricinus sp. & - & - & - & - & - & - & - & - & 2.76 & - & - & - \\
\hline Robinia sp. & - & - & - & - & - & - & - & - & - & 1.93 & - & - \\
\hline Rosa sp. & 4.38 & - & - & - & - & - & - & - & - & - & - & - \\
\hline Rosaceae & - & - & 0.99 & - & - & 4.38 & - & - & - & - & - & - \\
\hline Rubus sp. & - & - & - & - & - & - & - & - & 8.99 & - & - & - \\
\hline
\end{tabular}

Volume 5 Issue 5, May 2016 www.ijsr.net 


\section{International Journal of Science and Research (IJSR) \\ ISSN (Online): 2319-7064}

Index Copernicus Value (2013): 6.14 | Impact Factor (2015): 6.391

\begin{tabular}{|c|c|c|c|c|c|c|c|c|c|c|c|c|}
\hline Rutaceae & - & - & - & - & - & - & 8.06 & - & - & - & - & - \\
\hline Salix sp. & - & - & - & - & - & 5.85 & - & - & - & - & - & - \\
\hline Salvia sp. & - & - & - & - & - & - & - & - & - & 3.64 & - \\
\hline Sapindaceae & - & - & - & - & - & - & - & - & 2.80 & - & - \\
\hline Sapindus sp. & - & - & - & - & 3.25 & - & - & - & - & - & - \\
\hline Solaum sp. & - & - & - & 2.23 & - & - & - & - & - & - & - & - \\
\hline Sonchus sp. & - & - & - & - & - & - & - & 2.68 & 1.93 & - & - & - \\
\hline Syzygium sp. & - & - & 2.64 & - & - & - & 24.12 & 5.68 & 16.02 & - & - \\
\hline Taraxacum sp. & - & - & - & - & 2.09 & - & 1.05 & - & - & - & - & - \\
\hline Trifolium sp. & - & - & - & - & - & 16.11 & - & - & - & - & - \\
\hline Violaceae & - & - & - & 0.09 & - & - & - & - & - & - & - \\
\hline Vitis sp. & - & - & 1.93 & - & - & - & 0.62 & - & 1.99 & - & - & - \\
\hline Woodfordia sp. & 4.45 & - & - & - & 17.94 & - & - & - & - & - & - \\
\hline Zea sp. & - & - & - & - & - & - & - & - & - & 4.13 & - & - \\
\hline Zizyphus sp. & - & - & - & - & - & - & - & - & - & 5.06 & - & - \\
\hline
\end{tabular}

Percentage contribution of different plant species to honey samples from different areas of Shiwalik hills

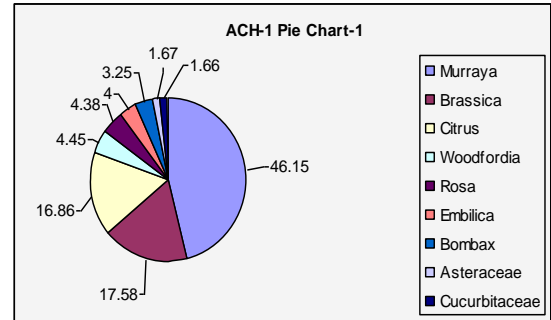

Figure A: Kumarhatti honey

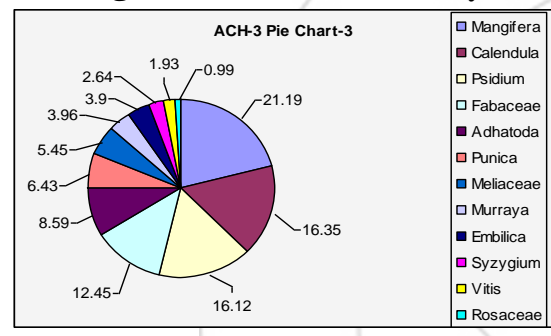

Figure C: Ropar honey

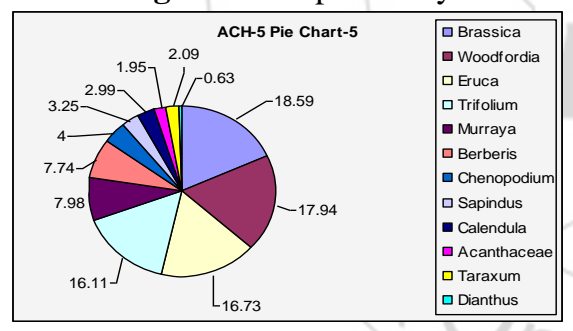

Figure E: Ramshahar honey

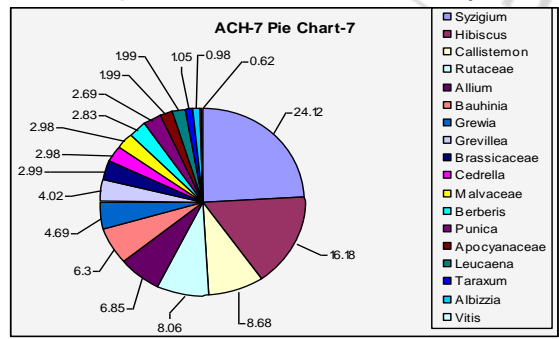

Figure G: Diggal honey

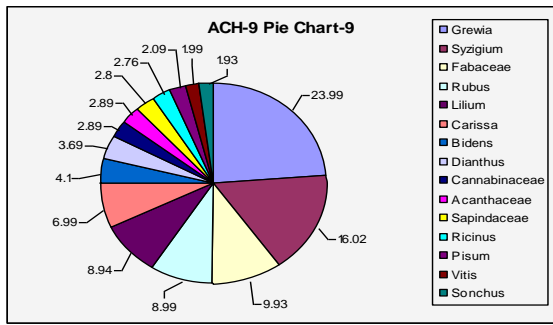

Figure I: Gagret honey

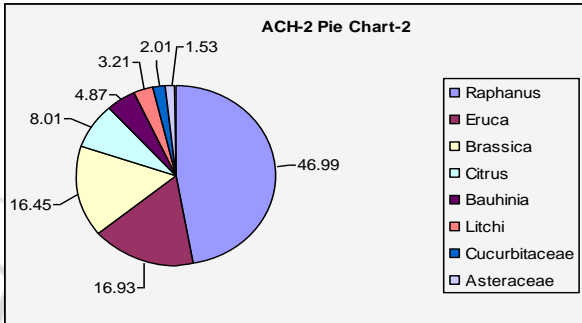

Figure B: Plasare honey

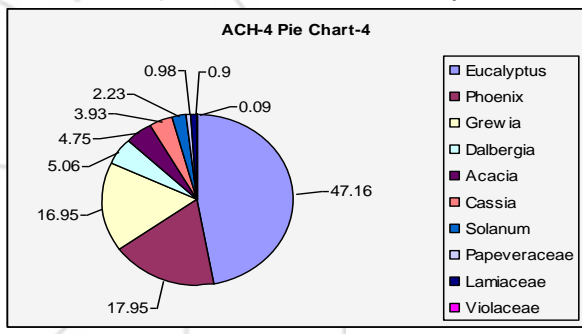

Figure D: Panchkula honey

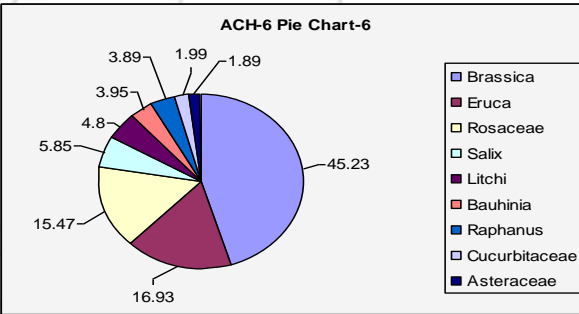

Figure F: Mittian honey

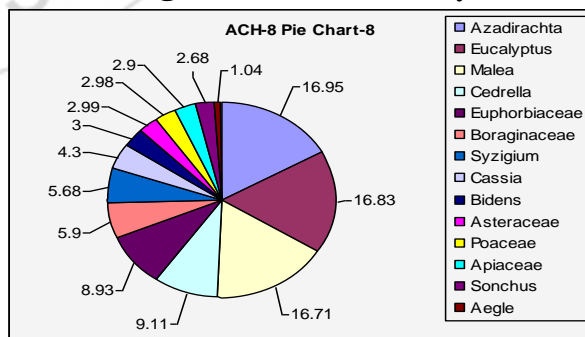

Figure H: Una proper honey

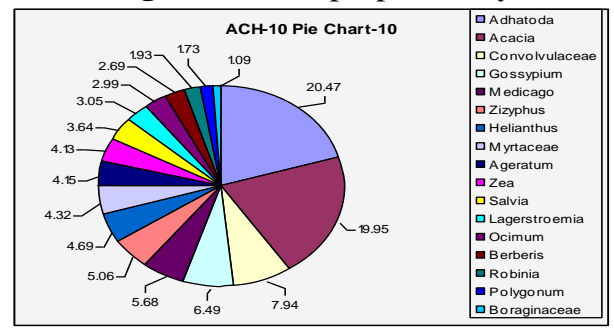

Figure J: Pinjore honey 


\section{International Journal of Science and Research (IJSR) \\ ISSN (Online): 2319-7064}

Index Copernicus Value (2013): 6.14 | Impact Factor (2015): 6.391

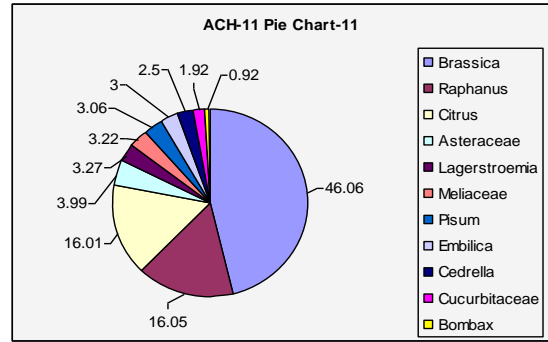

FigureK: Chandigarh honey

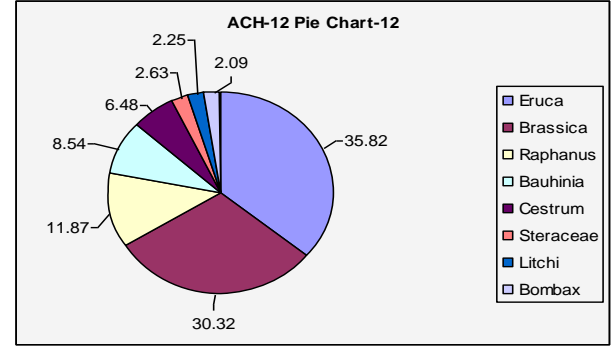

FigureL: Derabassi honey

Table 2: Pollen spectrum of honey samples of Apiscerana F. collected from different areas of Shiwalik hills

\begin{tabular}{|c|c|c|c|c|c|c|c|c|}
\hline Locality & Season & $\begin{array}{l}\text { Month of } \\
\text { Collection }\end{array}$ & $\begin{array}{l}\text { Colour of } \\
\text { honey }\end{array}$ & $\begin{array}{l}\text { Unifloral or } \\
\text { Multifloral }\end{array}$ & $\begin{array}{l}\text { Predominant } \\
\text { pollen type }\end{array}$ & $\begin{array}{l}\text { Secondary } \\
\text { pollen type }\end{array}$ & Important minor pollen types & Minor pollen types \\
\hline 1 & 2 & 3 & 4 & 5 & 6 & 7 & 8 & 9 \\
\hline Kumarhatti & Spring & April & $\begin{array}{l}\text { Light } \\
\text { amber }\end{array}$ & Unifloral & Murraya sp. & $\begin{array}{l}\text { Citrus sp., } \\
\text { Brassica sp. }\end{array}$ & $\begin{array}{l}\text { Bombax sp., Woodfordia sp., } \\
\text { Rosa sp., Embilica sp. }\end{array}$ & $\begin{array}{c}\text { Cucurbitaceae } \\
\text { Asteraceae }\end{array}$ \\
\hline Plasare & Winter & February & $\begin{array}{l}\text { Watery } \\
\text { white }\end{array}$ & Unifloral & Raphanus sp. & $\begin{array}{l}\text { Brassica sp., } \\
\text { Eruca sp. }\end{array}$ & $\begin{array}{l}\text { Bauhinia sp., Litchi sp., } \\
\text { Citrus sp. }\end{array}$ & Asteraceaecucurbitaceae \\
\hline Ropar & Summer & May & $\begin{array}{l}\text { Light } \\
\text { amber }\end{array}$ & Multifloral & & $\begin{array}{l}\text { Mangifera sp., } \\
\text { Psidium sp., } \\
\text { Calendula sp. }\end{array}$ & $\begin{array}{c}\text { Adhatoda sp., Murraya sp., } \\
\text { Meliaceae, Embilica sp., } \\
\text { Punica sp., Fabaceae }\end{array}$ & $\begin{array}{c}\text { RosaceaeSyzygium sp., Vitis } \\
\text { sp. }\end{array}$ \\
\hline Panchkula & Summer & June & $\begin{array}{l}\text { Light } \\
\text { amber }\end{array}$ & Unifloral & Eucalyptus sp. & $\begin{array}{c}\text { Phoenix sp., } \\
\text { Grewia } s p . \\
\end{array}$ & $\begin{array}{c}\text { Acacia sp., Dalbergia sp., } \\
\text { Cassia sp. }\end{array}$ & $\begin{array}{c}\text { Violaceae, Lamiaceae, } \\
\text { Papaveraceae }\end{array}$ \\
\hline Ramshahar & Summer & May & Amb & floral & & \begin{tabular}{|l|} 
Brassica sp., \\
Trifolium sp., \\
Woodfordia sp., \\
Eruca sp. \\
\end{tabular} & $\begin{array}{l}\text { Berberis sp., Sapindus sp., } \\
\text { Murraya sp., Chenopodium } \\
\text { sp. }\end{array}$ & $\begin{array}{c}\text { Taraxum sp., Dianthus sp., } \\
\text { Calandula sp., Acanthaceae } \\
\text { sp. }\end{array}$ \\
\hline Mittian & Winter & February & $\begin{array}{l}\text { Light } \\
\text { Amber }\end{array}$ & Unifloral & Brassica sp. & $\begin{array}{l}\text { Eruca sp., } \\
\text { Rosaceae }\end{array}$ & $\begin{array}{l}\text { Bauhinia sp., Raphanus sp., } \\
\text { Salix sp., Lithi sp. }\end{array}$ & Asteraceae, Cucurbitaceae \\
\hline Diggal & Summer & May & $\begin{array}{l}\text { Light } \\
\text { Amber }\end{array}$ & Multifloral & & $\begin{array}{l}\text { Syzygium sp., } \\
\text { Hibiscus sp. }\end{array}$ & $\begin{array}{l}\text { Grevillea sp., Rutaceae sp., } \\
\text { Allium sp., Bauhinia sp., } \\
\text { Grewia sp., Callistemon sp. }\end{array}$ & $\begin{array}{c}\text { Albizzia sp., Apocyanaceae, } \\
\text { Vitis sp., Brassicaceae, } \\
\text { Punica sp., Lucaena sp., } \\
\text { Malvaceae, Cedrella sp., } \\
\text { Taraxum sp. }\end{array}$ \\
\hline Una Proper & Summer & June & $\begin{array}{l}\text { Light } \\
\text { Yellow }\end{array}$ & Multifloral & & $\begin{array}{c}\text { Eucalyptus sp., } \\
\text { Melia sp., } \\
\text { Azadirchta sp. }\end{array}$ & $\begin{array}{l}\text { Syzygium sp., Cedrella sp., } \\
\text { Cassia sp., Euphorbiaceae, } \\
\text { Boraginaceae, Bidens sp. }\end{array}$ & $\begin{array}{c}\text { Aegle sp., Asteraceae, } \\
\text { Apiaceae, Sonchus sp., } \\
\text { Poaceae. }\end{array}$ \\
\hline Gagret & Summer & June & $\begin{array}{l}\text { Light } \\
\text { Amber }\end{array}$ & Multifloral & . & $\begin{array}{l}\text { Grewia sp., } \\
\text { Syzygium sp. }\end{array}$ & $\begin{array}{l}\text { Dianthus sp., Fabaceae, } \\
\text { Lilium sp., Rubus sp., Bidens } \\
\text { sp., Carissa sp. }\end{array}$ & $\begin{array}{c}\text { Acanthaceae, } \\
\text { Cannabinacee, Sonchus sp., } \\
\text { Vitis sp., Sapindaceae, } \\
\text { Pisun sp., Ricinus sp. }\end{array}$ \\
\hline Pinjore & Summer & July & & Multifloral & & $\begin{array}{c}\text { Adhadota sp., } \\
\text { Acacia sp. }\end{array}$ & $\begin{array}{l}\text { Medicago sp., Ageratum, } \\
\text { Zizyphus sp., Gossypium sp., } \\
\text { Myrtaceae, Helianthus sp., } \\
\text { Zea sp., Salvia sp., } \\
\text { Convolvulaceae, } \\
\text { Lagerstroemia sp. }\end{array}$ & $\begin{array}{c}\text { Berberis sp., Robinia sp., } \\
\text { Boraginaceae, Ocimum sp., } \\
\text { Polygum sp. }\end{array}$ \\
\hline Chandigarh & Spring & March & $\begin{array}{l}\text { Watery } \\
\text { white }\end{array}$ & Unifloral & Brassica sp. & $\begin{array}{l}\text { Raphanus sp., } \\
\text { Citirus sp. }\end{array}$ & $\begin{array}{l}\text { Pisum sp., Meliaceae sp., } \\
\text { Asteraceae sp., Embilica sp. }\end{array}$ & $\begin{array}{c}\text { Cedrellasp., Cucurbitaceae, } \\
\text { Bombaxsp. }\end{array}$ \\
\hline Derabassi & Winter & February & $\begin{array}{l}\text { Light } \\
\text { Yellow }\end{array}$ & Multifloral & & $\begin{array}{l}\text { Eruca sp., } \\
\text { Brassica sp. }\end{array}$ & $\begin{array}{l}\text { Raphanus sp., Bauhinia sp., } \\
\text { Cestrum sp. }\end{array}$ & $\begin{array}{c}\text { Litchi sp., Bombax sp., } \\
\text { Asteraceae. }\end{array}$ \\
\hline
\end{tabular}

Predominant pollen type $=45 \%$ and above Important minor pollen type $=3$ to $15 \%$

Secondary pollen type $=16$ to $45 \%$ and Minor pollen type $=<3 \%$

\section{Results}

The system adopted by Louveaux et al. (1970) was used for the presentation of frequencies of pollen grains in the honey samples. The following terms were used to describe the samples: 'predominant pollen grains' $(45 \%$ or more pollen grains present), 'secondary pollen grains' (16-45\%), 'important minor pollen grains' (3-15\%) and 'minor pollen grains' (less than 3\%). Honey samples having 45 or more grains of a single pollen type are termed 'unifloral honeys' and those having several pollen types in considerable percentage are termed 'multifloral honeys' (Iwama\&Melhem 1979; Chaturvedi 1983).
The pollen types found are shown in table 2:

Predominant pollen types (45\% or more): In the honey samples of Shiwalik hills, Murraya sp., Raphaumus sp., Eucalyptus sp., Brassica sp., were the predominant sporomorphs.

Secondary pollen types (16-45\%):- The following secondary pollen sources were present in honey; Citrus sp., Brassica sp., Mangifera sp., Psidium sp., Eruca sp., Calendula sp., Phoenix sp., Grewia sp., Trifolium sp., Woodfordia sp., Syzygium sp., Hibiscus sp., Eucalyptus sp., Melia sp., Azadirachta sp., Grewia sp., Adhatoda sp., Raphanus sp., and members of family Rosaceae.

Important minor and minor pollen types (up to 15\%):Pollen analysis of honey samples revealed Bombax sp., 


\section{International Journal of Science and Research (IJSR) \\ ISSN (Online): 2319-7064 \\ Index Copernicus Value (2013): 6.14 | Impact Factor (2015): 6.391}

Woodfordia sp., Rosa sp., Emblica sp., Bauhinia sp., Litchi sp., Citrus sp., Adhatoda sp., Murraya sp., Punica sp., Emblica sp., Acacia sp., Cassia sp., Berberis sp., Sapindus sp., Chenopodium sp., Bauhinia sp., Raphanus sp., Saix sp., Grevillea sp., Allium sp., Grewia sp., Callistemon sp., Cedrella sp., Syzygium sp., Bidens sp., Dianthus sp., Lilium sp., Rubus sp., Bidens sp., Carissa sp., Medicago sp., Ageratum sp., Zizyphus sp., Gossypium sp., Helianthus sp., Zea sp., Salvia sp., Pisum sp., Cesttrum sp., Bombax sp., Robinia sp., Ocimum sp., Albizzia sp., Vitis sp., Lucaena sp., Taraxacum sp., Aegle sp., Sonchus sp., Calendula sp., Dianthus sp., Ricinus sp., and members of families Cucurbitaceae, Asteraceae, Rosaceae, Violaceae, Lamiaceae, Papaveraceae, Acanthaceae, Apocyanaceae, Brassicaceae, Apiaceae, Cannabinaceae, Sapindaceae, Boraginaceae, Convolvulaceae, Meliaceae, Myrtaceae, Euphorbiaceae, Fabaceae, Rutaceae. Of the 12 honey samples 5 were unifloral and 7 were of multifloral origin.

\section{Discussion}

Present studies indicated that there are two honey flow seasons in Shiwalik hills, alternated by comparative dearth periods when there are floral gaps and to some extent bee colonies may experience nectar and pollen shortage. Present investigations suggest that different parts of Shiwalik hills have vast potential for the development of beekeeping due to multiplicity of bee flora available throughout the year. Pollen analysis also revealed a number of anemophilous pollen in different honey and pollen samples. They are Chenopodium sp., Zea sp., Psidium sp., members of families Euphorbiaceae and Poaceae.

\section{Conclusion}

Present melissopalynological and bee botanical investigations revealed that Centaureacyanus, Helianthus annus, Taraxacumofficinale, Bombaxceiba, Brassica spp., Eruca sativa, Raphanussativus, Carica papaya, Terminalia sp., Cucumis sp., Cucurbita sp., Acacia catechu, Dalbergiasissoo, Trifoliumalexandrinum, Trifoliumrepens, Woodfordiafructicosa, Moringaoleifera, Callistemon citrinus, Eucalyptus camaldulensis, Psidiumguajava, Syzygiumcumini, Sesamumindicum, Grevillea robusta, Eriobotrya japonica, Citrus spp., Salix babylonica, Litchi chinensis, Sapindusmukorosii, Camellia sinensis and Grewiaoptiva were major sources of pollen and nectar to honey bees in Shiwalik hills. Whereas, Adhatodavasica, Carissa caranda, Asclepiascurassavica, Cardusonopardiodes, Dahlia pinnata, Zinnia elegans, Impatiens glandiflora, Berberis spp., Brassica sp., Opuntia spp., Cannabis sativa, Benincasa spp., Embilicaofficinalis, Acacia arabica, Cassia fistula, Erythrinasuberosa, Delonixregia, Indigofera spp., Salvia spp., Lagerstroemia indica, Abelmoschusesculentus, Althaea rosea, Hibiscusrosa-sinensis, Malvaviscusarboreus, Epilobium spp., Clematis spp., Prinsepiautilis, Rubus spp., Murrayakoenigii and Antirrhinum majus were the medium plant resources.
Besides the above major and medium soruces other plant species which were identified as minor sources belonged to families Acanthaceae, Agavaceae, Amaryllidaceae, Anacardiaceae, Apiaceae, Arecaceae, Asteraceae, Bignoniaceae, Boraginacae, Caprifoliaceae, Chenopodiaceae, Convolvulaceae, Cucurbitaceae, Euphorbiaceae, Fabaceae, Fagaceae, Geraniaceae, Lamiaceae, Liliaceae, Linaceae, Malvaceae, Meliaceae, Maraceae, Musaceae, Papaveraceae, Plantiaginaceae, Poaceae, Polygonaceae, Portulacaceae, Punicaceae, Ranunculaceae, Rhamnaceae, Rosaceae, Rubiaceae, Solanaceae, Verbenaceae, Violaceae and Vitaceae.

Present studies indicated that there are two honey flow seasons in Shiwalik hills, alternated by comparative dearth periods when there are floral gaps and to some extent bee colonies may experience nectar and pollen shortage. Present investigations suggest that different parts of Shiwalik hills have vast potential for the development of beekeeping due to multiplicity of bee flora available throughout the year. For successful beekeeping floral calendars can prove to be excellent information sources of honey plants. This would be of great importance to bee keepers, extension of horticulture, agriculture and forest departments as its not only increases honey production but fruit quality is also greatly improved through such kind of apicultural investigations.

\section{Future Scope}

Assessment of bioresources including honey plant resources and their utilization pattern for hilly and plain area of North-West Himalayas. Floral maps should be prepared for whole of the Himalayan region. Detailed ecological and biochemical studies should be conducted on excellent nectar sources. Assessment of bioresources including honey plant resources and their utilization pattern for hilly and plain area of North-West Himalayas. For double-fold benefit, area based scientific beekeeping should be encouraged among the farmers. Need based research activities should be taken up.

\section{Reference}

[1] Chaturvedi, M. 1983. Pollen analysis of autumn honeys of Kumaon region. Proc. Indian Nat. Sci. Acad. 49: 125-133.

[2] Deodikar, G.B. 1965. Melittopalynology. Indian Bee J. 27: 59-72.

[3] Free, J.B. 1993. Insect Pollination of Crops. Academic Press, London.

[4] Iwama, S. and Melhem, T.S. 1979. The pollen spectrum of the honey of Tetragoniscaangustulaangustula L. Apidologie 10: 275-295.

[5] Louveaux, J.; Maurizio, A. and Vorwohl, G. 1978. Methods of melissopalynology. Bee World 59: 139157.

[6] McGregor, S.A. 1976. Insect pollination of cultivated crop plants. USDA/ARC Agric. Washington, DC handbook, 496 pp. 


\section{International Journal of Science and Research (IJSR) \\ ISSN (Online): 2319-7064}

Index Copernicus Value (2013): 6.14 | Impact Factor (2015): 6.391

[7] Nair, P.K.K. 1985. Melittopalynology. In Essencials of Palynology. Today and Tomorrow's Publishers, New Delhi, 59-64 pp.

[8] Seeley, T.D. 1985. Honeybee Ecology - A Study of Adaptation in Social Life. Princeton Univ. Press, Princeton.

[9] Sharma, N. 1989. Melissopalynology and survey of honey plants in Himachal Pradesh. Ph.D. Thesis: Himachal Pradesh University, Shimla, India.

[10] Suryanarayana, M.C. 1978. Bee plants of India, Carviacallosa. Indian Bee J. 40: 7-10.

\section{Author Profile}

V. K. Mattu is Professor at Department of Biosciences, Himachal Pradesh University Shimla

Avinash Kaur is a Ph.D. Research Scholar at Department of Biosciences, Himachal Pradesh University Shimla. 